\title{
Quantitative analysis of liver tumors at different stages using microscopic hyperspectral imaging technology
}

Jiansheng Wang

Qingli Li 


\title{
Quantitative analysis of liver tumors at different stages using microscopic hyperspectral imaging technology
}

\author{
Jiansheng Wang ${ }^{\mathrm{a}}$ and Qingli $\mathrm{Li}^{\mathrm{a}} \mathrm{b}, \mathrm{k}$ \\ ${ }^{a}$ East China Normal University, Shanghai Key Laboratory of Multidimensional Information Processing, Shanghai, China \\ ${ }^{\mathrm{b} E n g i n e e r i n g ~ C e n t e r ~ o f ~ S H M E C ~ f o r ~ S p a c e ~ I n f o r m a t i o n ~ a n d ~ G N S S, ~ S h a n g h a i, ~ C h i n a ~}$
}

\begin{abstract}
Liver cancer has one of the highest rates of human morbidity and mortality. However, in terms of pathology, liver cancer is traditionally clinically diagnosed based on observation of microscopic images of pathological liver sections. This paper investigates in vitro samples of rat models of bile duct carcinoma and presents a quantitative analysis method based on microscopic hyperspectral imaging technology to evaluate liver cancers at different stages. The example-based feature extraction method used in this paper mainly includes two algorithms: a morphological watershed algorithm is applied to find object and segment pathological components of pathological liver sections at different stages, and a support vector machine algorithm is implemented for liver tumor classification. Majority/minority analysis is utilized as the postclassification tool to eliminate small plaques from the preliminary classification results. Then, pseudocolor synthesis in RGB color space is used to produce the final results. The experimental results show that this method can effectively calculate the percent tumor areas in liver biopsies at different time points, that is, $3.338 \%, 11.952 \%, 15.125 \%$, and $23.375 \%$ at $8,12,16$, and 20 weeks, respectively. Notably, through tracking analysis, the processed results of 8-week images showed the possibility for early diagnosis of the liver tumor. @ 2018 Society of Photo-Optical Instrumentation Engineers (SPIE) [DOI: 10.1117/1 JBO.23.10.106002]
\end{abstract}

Keywords: pathological evaluation; microscopic hyperspectral imaging; morphological watershed algorithm; support vector machine; liver tumor.

Paper 180220RRR received Apr. 15, 2018; accepted for publication Sep. 13, 2018; published online Oct. 1, 2018.

\section{Introduction}

According to the latest statistics from the China National Cancer Center, liver cancer has become the third most fatal cancer. The incidence of liver cancer gradually increases with age, especially after age 40, and males outnumber females. Unfortunately, the 5 -year survival rate for liver cancer is only $\sim 10.1 \%$, posing a severe threat to human health. Generally, bile duct carcinoma is a common primary liver malignancy, which occurs largely in patients with a chronic liver disease, such as cirrhosis. The pathological appearance of liver tumors is abnormal liver cells arranged on typical trabecular structures, and large liver tumor lesions usually have hemorrhage and fibrosis. Due to the complex pathogenesis of liver tumors and difficult-tocontrol high-risk factors, liver tumors are challenging to prevent. At the same time, less-effective screening techniques and lower early diagnosis techniques cause liver tumors to be generally late when discovered. Consequently, reducing the incidence, improving the early diagnosis rate, increasing the survival rate, and promoting homogenization are the focus of the medical community and the research field on the prevention and control of liver tumors.

Modern medical imaging plays a vital role in liver tumor diagnosis. Chauvie et al. ${ }^{1}$ validated a fully automated approach for the liver uptake measurement in whole-body fluorodeoxyglucose positron emission tomography/computed tomography (CT) scans and extracted its average standardized uptake value. Kim et al. ${ }^{2}$ described the imaging characteristics of

*Address all correspondence to: Qingli Li, E-mail: qlli@cs.ecnu.edu.cn primary hepatic angiosarcomas on gadoxetate disodiumenhanced dynamic magnetic resonance imaging and highlighted features that help distinguish angiosarcomas from hemangiomas of similar size. In recent years, digital subtraction angiography (DSA) has been developing, combined with CT techniques for liver tumor examination. Song et al. ${ }^{3}$ proposed a hypothetical anatomic model to identify and classify the broad spectrum of celiac axis and common hepatic artery variations in the use of both spiral CT and DSA in a large study population. Most imaging methods are noninvasive but are limited by the hardware device and software technology in the early detection rate of liver tumors. Although hepatic arteriography often allows for more accurate and rapid diagnosis, it is an invasive examination that is considered for use only when other examinations cannot be used for accurate evaluation and diagnosis.

Researchers currently tend to analyze histopathology image of pathological liver sections, which can provide liver cell morphological characteristics and lesion texture information. Shimada et al. ${ }^{4}$ researched macroscopic and microscopic indocyanine green (ICG) fluorescence imaging characteristics of normal liver and liver tumors using ICG fluorescence method, confirming that ICG fluorescence can also be used for postoperative pathological examination of microscopic lesions of pathologic liver tissue specimens. Liver fibrosis is a common pathological process of chronic liver tumors. Zhou et al. ${ }^{5}$ applied Mueller matrix microscopy to human liver fibrosis at different stages of hepatic fibrosis to extract affected areas for quantitative analysis. They also verified the validity of the experimental 
results with the Monte Carlo simulation method. With the continuous advancement of computer-assisted technology, the role of liver tissue pathological image classification in clinical diagnosis has become more and more significant. Ashour et $a .^{6}$ extracted features of granuloma stages, namely cellular, fibrocellular, and fibrotic granulomas along with normal liver samples and then classified. They proposed a new hybrid combination of statistical features for early prediction and treatment with empirical mode decomposition. Moraru et al. ${ }^{7}$ classified healthy, cellular, fibrocellular, and fibrous liver pathologies based on the liver state, and gradient vector and gradient angle co-occurrence matrix approaches were used to identify abnormal tissue structures. Their experimental results confirmed that local anisotropy-based texture measurement is more productive for microscopic pathological analysis. Recently, collagen proportional area (CPA) extraction in liver biopsy images has supplied an effective means for the assessment of fibrosis in liver tissue. ${ }^{8,9}$ Tsipourasa et al. $^{9}$ utilized clustering algorithms, $\mathrm{K}$-means (KM), and fuzzy C-means for liver tissue separation, and six different classifiers were employed for regional classification. Tsouros et al. ${ }^{10}$ proposed a modification of the $\mathrm{K}$-means clustering algorithm, namely supervised restriction of centroid movement for image segmentation of liver biopsies. However, the lack of standardized methods for computer image analysis for CPA assessment limits the application of CPA in clinical practice. A lot of researchers have presented and confirmed a series of possible liver tumor analysis means via microscopic image, but the two-dimensional (2-D) images generally only include spatial and texture characteristics. For liver tumor prediagnosis, the traditional optical microscope image identification has limitation to some extent.

Microscopic hyperspectral imaging (MHSI) as a new type of medical image processing technology opens up a higher-efficiency field for the histopathological diagnosis. Hyperspectral imaging originates from remote sensing imaging and contains abundant electromagnetic spectra. ${ }^{11}$ Even in the same biological tissue, absorption and reflectance spectra by different structures and components are diversified. Therefore, hyperspectral imaging can be considered to identify and diagnose pathological tissue in medical images. Jarman et al. ${ }^{12}$ built a hyperspectral microscope to capture focused and intensity corrected images with wavelength ranging from 450 to $750 \mathrm{~nm}$ with $\sim 10-\mathrm{nm}$ spectral resolution and submicron spatial resolution. They showed the presence of different components from a nonabsorbent saliva droplet sample. Neittaanmaki-Perttu et al. ${ }^{13,14}$ proved the feasibility of a hyperspectral imaging system (HIS) in the early detection of field cancerization with a new, noninvasive method. Furthermore, they showed the potential of HIS in the detection of the subclinical borders of lentigo maligna (LM) and lentigo maligna melanoma and confirmed histologically applications. Hashimoto et al. ${ }^{15}$ classified HE-stained liver pathology hyperspectral images based on the bag of features, and the average classification accuracy of different tissue structures had improved. Overall, there have been few studies on the autodetection of cancerous liver tissue at different stages, and that analyze and quantify the pathological changes with microscopic hyperspectral images for early diagnosis of liver tumors.

A large number of research studies have shown that features extraction is of great significance for liver tumor classification. Therefore, this paper introduced a statistical features extraction of liver tumor using MHSI images of bile duct carcinoma animal models (rats) at different stages, aiming to explore the feasibility of early pathological diagnosis of liver tumors. The original microscopic hyperspectral image needs preprocessing for further image analysis. Morphological watershed algorithm (MWA) is applied to segment grayscale image of liver pathological slice into numerous regions; then support vector machine (SVM) is utilized as a supervised classification algorithm to integrate the spatial, textured, and spectral information to identify the liver tumor from the background based on example selections. To achieve accurate statistics for the percent tumor area in liver samples, the majority/minority analysis (MMA) technique is performed on the classification results. Compared with the SVM method, the classification accuracy of MWAbased SVM is higher. Further, pseudocolor synthesis is used to highlight the form and location of liver tumors in tissue sections. The results indicate that the percent tumor area in the liver tissue sections is positively related to the length of the experimental period. Moreover, the analytical results at 8 weeks serve as a valuable reference for the prediagnosis of liver tumors.

\section{Materials and Methods}

The spectral measurements of a large number of substances indicate that different objects exhibit different spectral reflectance and radiation characteristics, which cause the wavelength width of the absorption peaks and reflection peaks to be $\sim 5$ to $50 \mathrm{~nm}$. Its physical connotation is the lattice vibrations of different molecules, atoms, and ions, which cause spectral emission and absorption of different wavelengths, thus different spectral characteristics are produced, so the spectral characteristics can be used to analyze and identify the tissue components. ${ }^{16}$ Medical pathological images are commonly treated with stains. Although the stains have an influence on the spectral characteristics of biological tissues, the spectral characteristics of different components may also differ after staining, ${ }^{17}$ which has a negligible interference on the feature extraction to a certain extent. In our work, the liver tumor feature extraction and statistical analysis method proposed can be summarized with a flowchart shown in Fig. 1. The details are elucidated in the following sections.

\subsection{Hyperspectral Images Acquisition}

Hyperspectral imaging combines imaging technology with spectroscopy to detect 2-D spatial and one-dimensional (1-D) spectral information of a target and obtain continuous and narrowband image data with high spectral resolution. In this paper, microscopic hyperspectral images of pathological liver sections were acquired through an acousto-optic tunable filter (AOTF)-based molecular hyperspectral imaging platform. The system has six parts: a microscope (Nikon 80i, Nikon Corp.), a radiofrequency driver [specific pathogen free (SPF) model AOTF controller, VFI-138.5-93-SPS-A-C2, Brimrose Corp.], an AOTF adapter (CVA200-0.55-1.0-L, Brimrose Corp.), a 1/1.8-in. high-density cooled charge-coupled device (CCD) camera (DS-2MBWc, Nikon, Japan), a data collection and control module (Camera Control Unit DS-U2, Nikon Corp.), and personal computer software and hardware. ${ }^{11,18,19}$ The light source integrated with the microscope is a $120-\mathrm{W}$ halogen lamp (Epi-illumination, Eclipse 80i, Nikon Corp.), which is a crucial part that provides illumination for the entire imaging system. To test the detection performance of the system at different wavelengths, a blank slide with no samples is placed on the microscopy stage. The microscopy light source is turned on and microscopic hyperspectral image data are captured. Then, the 


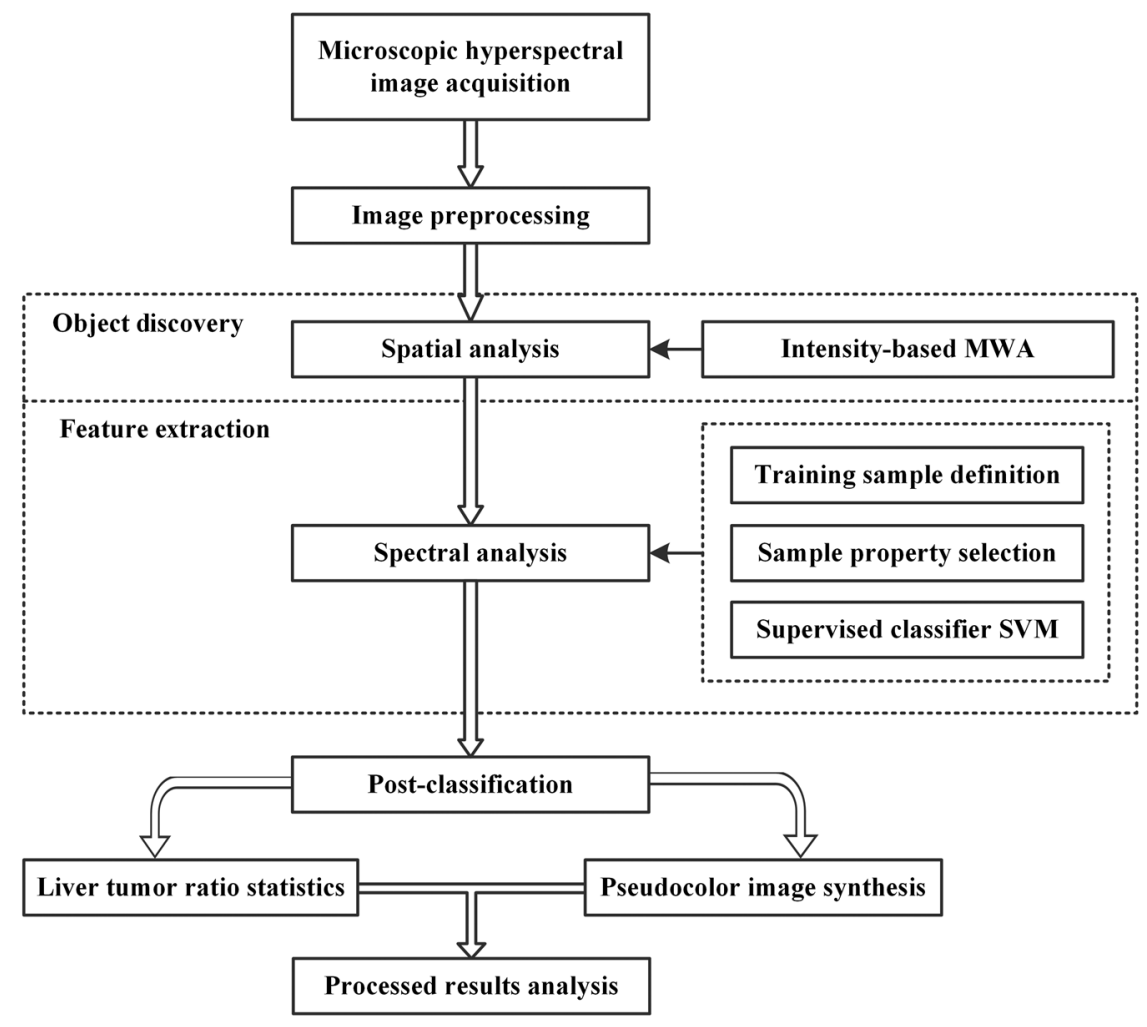

Fig. 1 The flowchart of the proposed method.

spectra of different pixels are extracted from the blank MHSI image data, and the averaged spectrum is calculated (as shown in Fig. 2), which can be used to represent for the overall spectral response of the system. The ordinate of Fig. 2 is the gray value, that is, the measured value ( 2 bytes) of the system detector at different wavelengths. The spectral range of the halogen lamp is relatively wide, which is conducive to the detection of each wavelength of hyperspectral imaging at 550 to $1000 \mathrm{~nm}$. The AOTF adapter with a $10-\mathrm{mm} \times 10-\mathrm{mm}$ aperture is one of the core components, providing a narrow bandwidth. The reflected or transmitted light acts as a carrier for the spatial and spectral information after interacting with the pathological tissues of the live, captured, filtered by the AOTF adapter and imaged on the CCD detector. Computer software and hardware are used to control the data acquisition of the MHSI system,

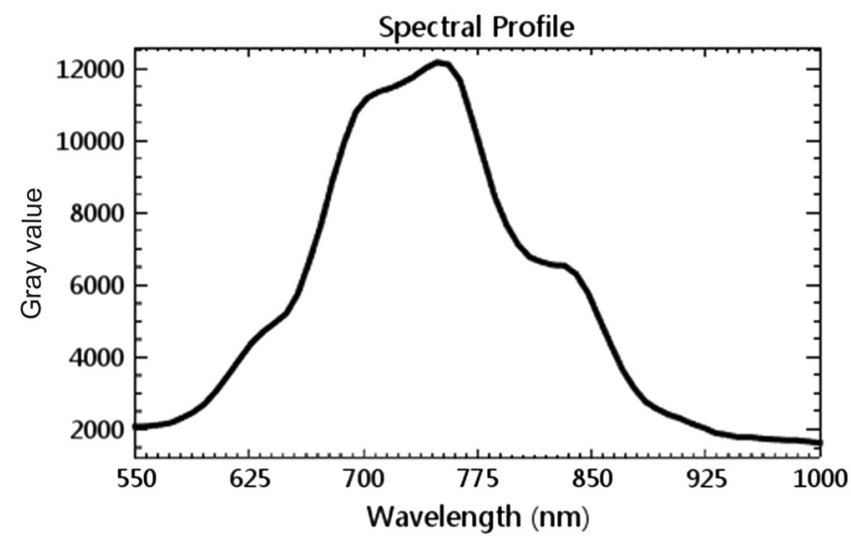

Fig. 2 The overall spectral response of the system (including the factor of the halogen lamp). process and analyze the datasets, and provide storage space for the hyperspectral images.

The real spatial resolution of the MHSI system depends on the pixel size of the CCD detector and the optical modulation transfer function of the system. ${ }^{18}$ To evaluate the practical spatial resolution of the system, a microscope graticule $(\mathrm{C} 1$, Shanghai Jinnshine Photonics Technology Co., Ltd.) was used as a standard sample and observed by the MHSI system. The graticule is a 1-mm scale divided into 100 divisions, where each small division is $0.01 \mathrm{~mm}$. The Nikon CCD is a monochrome camera that features a 2 megapixel detection array $(1600 \times 1200$ pixels $)$, high sensitivity with pixel size of $4.4 \times 4.4 \mu \mathrm{m}$, and high quantum efficiency. According to the graticule images captured by the MHSI system, the spatial resolution of the system is $<1 \mu \mathrm{m}$, which is suitable for pathological analysis. Figure 3 shows a typical hyperspectral image of a liver tissue section used in this study. The image is rendered as an $n$-dimensional data cube (a), containing 60 wavebands of spectral information. Each waveband of the hyperspectral image consists of $1280 \times 1024$ pixels $\times 12$ bits $/$ pixel, the wavelength range $(b)$ is from 550 to $1000 \mathrm{~nm}$, and the spectral resolution is 2 to $6 \mathrm{~nm}(2 \mathrm{~nm}$ at $543 \mathrm{~nm} ; 6 \mathrm{~nm}$ at $792 \mathrm{~nm})$. Once acquired, the dataset is stored in the band sequential file format.

\subsection{Image Preprocessing}

The original MHSI images cannot be directly used for image segmentation due to insufficient accuracy. Hence, two main image preprocessing procedures, spectral domain normalization and spatial domain enhancement need to be performed on the original images.

Affected by the transmission process of the microscope light source and the degree of liver pathological section cleanliness, 


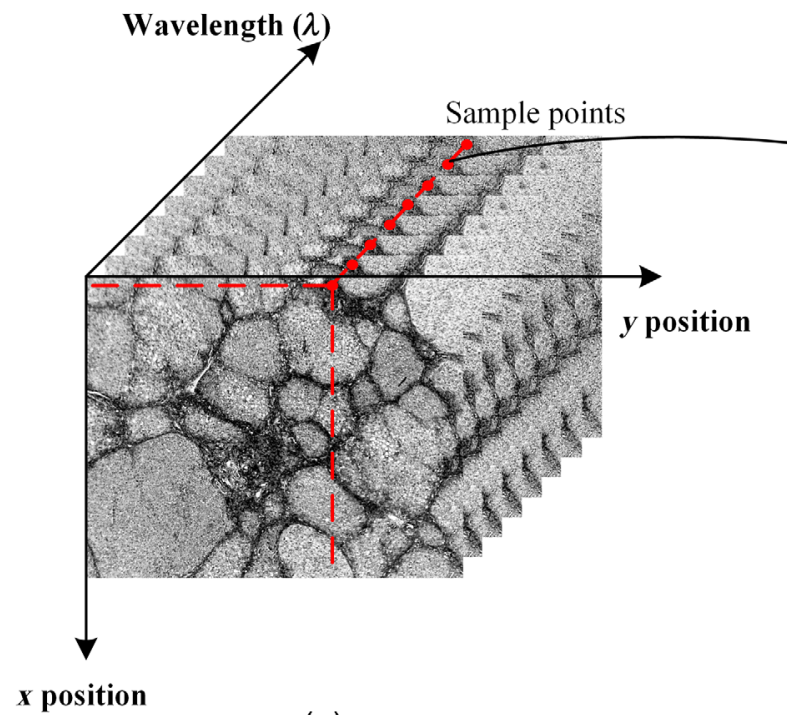

(a)

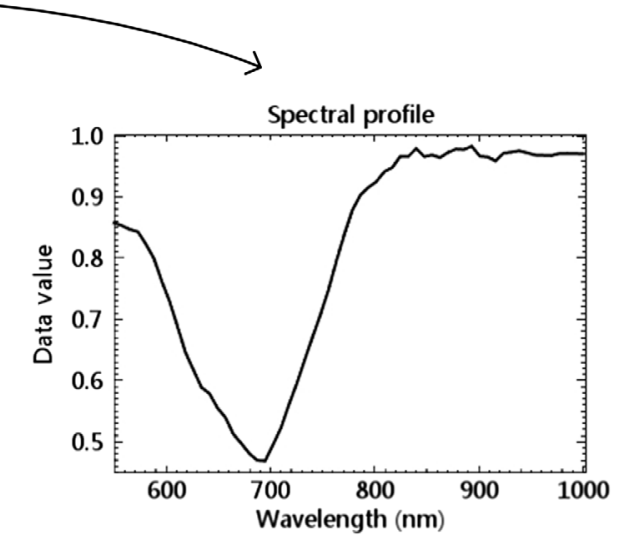

(b)

Fig. 3 Liver biopsy MHSI data cube. (a) The data cube model and (b) the spectral curve in the range of 550 to $1000 \mathrm{~nm}$.

the original image embodies artifacts from the data and abnormal noise points. Spectral-domain normalization achieves the spectral response calibration to obtain the relative (or percent) transmittance instead of the absolute intensity data for further data analysis, and this process eliminates image artifacts and some noises. When collecting liver tissue section images treated with stains, a blank reference image corresponding to the same glass slide is also collected, and then spectral math tool is utilized to the two images. This process is defined as the following equation:

$$
\begin{aligned}
& S_{n}^{\text {output }}=\operatorname{float}\left(S_{n}^{\text {sample }}\right) / \text { float }\left(S_{n}^{\text {blank }}\right) \\
& n \text { from } 550 \text { to } 1000(\mathrm{~nm}),
\end{aligned}
$$

where $n$ is the $n$ 'th wavelength of the hyperspectral image. $S_{n}^{\text {sample }}$ and $S_{n}^{\text {blank }}$ represent the value of spectrum before calibration, respectively, and float expresses data-type conversion functions. $S_{n}^{\text {output }}$ is the spectral value of the output image in the $n$ 'th wavelength after normalization. Figure 3(b) shows the result of spectral-domain normalization.

Hyperspectral images of the pathological liver section include impulse noise, hardware equipment such as a CCD detector, and software operation such as decoding process will produce lots of salt and pepper noise, which is common in digital images. ${ }^{20}$ The composition of the pathological liver tissue is complicated, and the black-and-white noise points in hyperspectral images interfere with image processing. Median filtering is an effective way of spatial-domain enhancement to remove the isolated noise and further improve the visual effect. The math equation of the median filter can be given as follows:

$y_{i, j}=\operatorname{Med}_{A}\left\{x_{i, j}\right\}$,

where $A$ is the convolution kernel size, $x_{i, j}$, for $(i, j) \in I \equiv$ $\{1, \ldots, M\} \times\{1, \ldots, N\}$, is the gray level of a true $M \times N$ image $x$ at the pixel location $(i, j)$, and $y_{i, j}$ is the output matrix. The research on median filtering has been quite mature. In this step, we want to improve the image visual quality under the premise of retaining the liver tumor pixels to the maximum extent. The parameter $r$ is defined as the noise level, ${ }^{21}$ after spectral domain normalization, $r$ of the image is much $<25 \%$; therefore, the filter window size is set to $3 \times 3$.

\subsection{Spatial Analysis: Grayscale Image Segmentation}

The universal hyperspectral image analysis methods use its abundant spectral information for image segmentation but lack information about the spatial structure of the image. For the hyperspectral images of pathological liver sections, tumor lesions cannot be differentiated easily by the human eyes, particularly in the 8-week images, it is arduous to distinguish the lesion location directly. Spatial information needs to be integrated into the image processing for the purpose of improving image segmentation performance. This paper uses a morphological watershed segmentation algorithm that extends liver tissue hyperspectral images to define information about the spatial structure. The standard watershed algorithm was proposed by Vincent and Soille at PAMI in $1991,{ }^{22}$ which has also been widely using in hyperspectral image segmentation. ${ }^{23-25}$ The watershed algorithm addresses the spatial information of 2-D images, and it does the same processing for each wavelength of hyperspectral images. Figure 4 is a representation of morphological watershed about the hyperspectral image in one wavelength presented by Tarabalka et al. The gray level of each pixel in the image represents the elevation of the point. Each local minimum value and its affected area is called a catchment basin, whereas the boundary of the catchment basins forms a watershed.

The MWA plays a prominent part in the gradient threshold segmentation of hyperspectral grayscale images of liver pathology slices. Several related auxiliary parameters are set as follows:

(i) An intensity-based algorithm is suitable for the segmentation of small gradient changes, for the probability of 


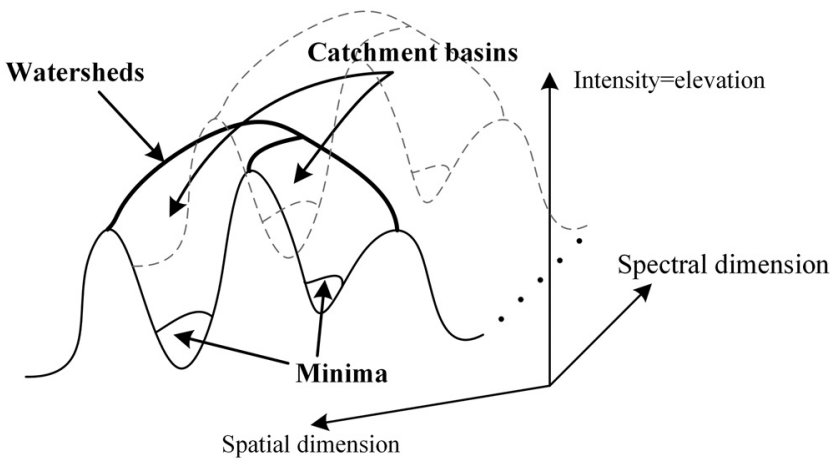

Fig. 4 Morphological watershed representation in one wavelength.

over-segmentation is smaller, with no need for merging algorithms, the segmentation can achieve ideal results.

(ii) In view of the complexity of liver tissue grayscale images, the segmentation threshold is located at $5 \%$ in this exam. The range of the segmentation threshold is between 0 and 100. The larger the scale is, the less number of areas the image segmentation will produce.

(iii) The texture kernel size is defined as 11 . In virtue of a larger size fits liver pathological images that have more extensive data area and less texture difference. The range of this parameter is 3 to 19 . We have chosen the mean of the maximum and minimum values after repeated experiments.

\subsection{Spectral Analysis: Liver Tumor Identification}

The quality of image segmentation determines the accuracy of the classification effect to a certain extent. A large number of repeated experiments manifested that the segmentation algorithm mentioned above selected the desired segmentation threshold and can segment the edge features of the liver tissue grayscale as well as possible. Currently, SVM is one of the most popular classification methods in hyperspectral images. $^{26,27}$ SVM can be defined as a linear classifier with the most substantial interval in feature space, and the learning goal of the linear classifier is to find a hyperplane in $n$-dimensional data space. ${ }^{28}$ The intuitive distance from the point to the hyperplane is expressed as a geometrical margin. However, most of the complex data are not linearly separable, for the nonlinear case, the processing method is to select a kernel function and solve the problem of linear inseparability in the original space by mapping the data to the high-dimensional space. In the spectral analysis of liver tumor classification, the Gaussian kernel function is chosen as the SVM kernel, which is an optimal radial basis function because of its excellent high-dimensional mapping capability. Its basic mathematical equation is

$K\left(x-x_{c}\right)=\exp \left(-\frac{\left\|x-x_{c}\right\|^{2}}{2 \sigma^{2}}\right)$,

where $x$ is a feature and $x_{c}$ is a landmark, $\sigma^{2}$. is the parameter of the Gaussian kernel function to adjust the speed of the descent, in this step $\sigma^{2}$ takes a value of 1 . The Gaussian kernel function measures the similarity by measuring the distance between $x$ and $x_{c}$ (the closer it shows the more similar and the similarity is closer to 1 , the farther it shows the less similar and the similarity is closer to 0). In the experiments, the empirical value 1 is used to get better results. This process maps out new features $K\left(x-x_{c}\right)$.

To classify the liver tumors more accurately, the examplebased SVM method was adopted for image classification, and there are some operations that need to be executed.

(i) Defining training samples: We have sampled and created the spectral libraries of liver tumors and normal tissues by selecting regions of interest under the guidance of pathologists. ${ }^{29}$ Using the segmentation results obtained by the MWA, we selected a subset of liver tumor and normal tissue region as classified reference examples.

(ii) Selecting sample properties: Different attribute selections are beneficial to train the classification examples. In the experiment, spectral mean (average grayscale value at selected wavelength), spectral maximum and minimum (maximum and minimum grayscale values at selected wavelength), and spectral standard deviation (standard deviation of grayscale value at selected wavelength) for each wavelength are calculated, and all wavelengths are combined in the SVM classification process. Another attribute is the texture. The convolution operation is performed using the texture kernel size set in the watershed segmentation algorithm. The value of the center pixel of the window is replaced with the acquired attribute value, and then the attribute value with the same tag value in the image segmentation result is averaged.

\subsection{Postclassification and Evaluation}

The application of supervised classification methods will inevitably produce some very small-area patches, which are usually similar to tumor spectrum but nonpathological components in liver tissue. Preliminary classification results by MWA-based SVM algorithm are kept for further classification processing, namely employing MMA tools to reject or reclassify the small plaques. The majority analysis defines a transform kernel size using a convolution filtering measure to replace the central pixel class with the dominant pixel class (the largest number of pixels) in the transform kernel, and it is suitable for postclassification of liver hyperspectral images. In this section, the transform kernel size is set as $9 \times 9$, and the center pixel weight is controlled at 1 to improve the accuracy of small plaque removal in liver tissue images, and the processed images are the final classification results. Synchronously, we only used the SVM algorithm to do the tumor recognition. In the course of the experiment, the pathologist provided us with the standard classification images, and we made a comparative analysis of the three results.

To evaluate the accuracy of the proposed method, two indices by means of the confusion matrix are used to evaluate and compare the classification results: overall accuracy (OA) and Kappa coefficient. The confusion matrix ${ }^{30}$ is used to compare the classification result with the standard image, by comparing the position and classification of each original pixel with the corresponding position and classification in the classification image. In this paper, the OA is defined as follows: 
Overall accuracy

$$
=\frac{\sum \text { True Positive }(\mathrm{TP})+\sum \text { True Negative }(\mathrm{TN})}{\sum \text { Total Pixels }},
$$

where TP represents a correctly classified liver tumor pixels, and TN represents a correctly classified normal tissue pixel. Total pixels are provided by the standard classification image. The OA only considers the number of pixels correctly classified in the diagonal direction, whereas the Kappa coefficient considers various missing and misleading pixels outside the diagonal. This index can calculate overall consistency and classification consistency. The kappa coefficient is computed as follows:

$K=\frac{N \sum_{i=1}^{r} p_{i i}-\sum_{i=1}^{r}\left(p_{i+} * p_{+i}\right)}{N^{2}-\sum_{i=1}^{r}\left(p_{i+} * p_{+i}\right)}$,

where $N$ is the total pixels of the standard image, $r$ is the number of rows for the confusion matrix, $\sum_{i=1}^{r} p_{i i}$ is the sum of the diagonal probabilities, and $\sum_{i=1}^{r}\left(p_{i+} * p_{+i}\right)$ is the sum of the products of the $K$ marginal probabilities. ${ }^{31}$ The OA combined with Kappa coefficient is more accurate for the classifier evaluation. We obtained two sets of data: SVM classification results compared to standard classification images and MWA-based SVM classification results compared with standard classification images. The results are discussed in the following sections.

\subsection{Statistical Analysis and Pseudocolor Synthesis}

The liver pathologic section images produced via MHSI are processed by the above segmentation and classification algorithms. Next, the percentage of pixels containing tumor tissue from whole images is calculated according to the final classification results. The statistical data at the four different time points are used for quantitative analysis of liver tumors.

The resolution of human eyes for color images is much higher than that of gray-scale images. ${ }^{32}$ Sammouda et al. ${ }^{33}$ have proven that color images in the RGB color space are ideal compared with HSV and HLS. Pseudocolor synthesis as a valid image enhancement method can highlight the morphology and distribution of a specific target. The purpose of this part is to enhance the information of pathological liver components, and the final effect of wavelength combination is to highlight the liver tumor from the image information. The size of singlewaveband information is not the dominant factor in determining the composition of the wavelength, and the high contrast between the liver tumor information and the normal area information is the focus of the way. Therefore, a pseudocolor synthesis method based on wavelength combination will act on the classification results, and waveband combination coefficient, RGB synthesis principle, and visual sensory judgment are adapted to select and verify the wavelength combination scheme.

For a sample image, the RGB three-composition has a strong correlation. In the experiment, the mentioned above classification result saved in the gray-scale format is selected as one component of RGB pseudocolor image synthesis; the remaining two components need to be selected by virtue of the superior spectral characteristics of the hyperspectral image. Determine the best wavelength combination based on the amount of information, namely, the maximum difference in standard deviation and the minimum sum of correlation coefficients between the combined wavebands. Take one of the experimental images for 20 weeks as an example. Figure 5(a) shows the standard deviation of a liver pathological microscopic hyperspectral image. The vertical axis illustrates the continuum removed value, where the actual spectral reflectance is used to remove the reflectance value of the corresponding wavelength on the envelope. It can eminently stress the absorption and reflection properties of the spectral curve, normalize them to consistent spectral values, and extract spectral features from these values. The process suppresses noise, and is useful for displaying characteristic values of the spectral curve. The peak and valley of the standard deviation appear approximately 700 and $900 \mathrm{~nm}$, respectively. The distribution of correlation coefficients in the range of 0 to 1 for the 60 wavebands (wavelengths from 550 to $1000 \mathrm{~nm}$ ) is shown in Fig. 5(b), and the first row and column of the chart are arranged in the order of wavelengths, respectively. The shaded area, whose initial wavelength is approximately at $770 \mathrm{~nm}$ (the 30th waveband), indicates that the waveband correlation coefficient is not $>0.5$ (ignore the waveband autocorrelation coefficient). This section combines the above two parameters and chooses the appropriate wavelength combination for image pseudocolor synthesis and for achieving the best visual contrast.

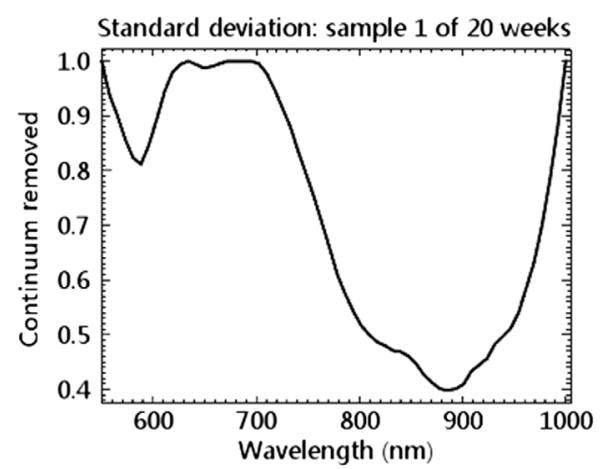

(a)

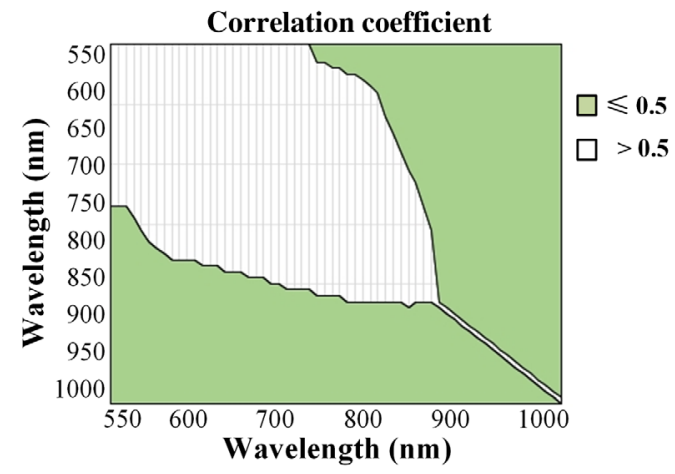

(b)

Fig. 5 Standard deviation and correlation coefficient distributions. (a) Standard deviation based on continuum removed value of liver pathological MHSI image and (b) correlation coefficient distribution of wavelength. 


\section{Experiments and Results}

\subsection{Experimental Data and Preprocessing}

In this work, 6-month-old SD (Sprague Dawley) male rats were purchased from Shanghai SLACCAS Experimental Animal Co., Ltd. [Animal Production License Number: SCXK (Shanghai) 2012-0002, Use License: SYXK (Shanghai) 2012-0002], reared in Tongji University affiliated Oriental Hospital Experimental Animal Center. Thioacetamide (TAA) was purchased in Changzhou Hangyu Pharmaceutical Technology Development Co., Ltd. (CAS No.27366-72-9). To establish a TAA-induced bile duct carcinoma rat model, $40 \mathrm{SD}$ male rats in the experimental group were selected, daily drinking water containing TAA concentration of $300 \mathrm{mg} / \mathrm{L}$, reared in an SPF animal house. Respectively, in feeding 8th, 12th, 16th, and 20th week, 10 rats were euthanized, the livers were harvested by surgery and paraffin-embedded, fixed, and stained with H\&E (hematoxylin-eosin staining) to make pathological sections. Synchronously, four rats in the control group were selected to drink purified water every day and kept in the same animal house. At each stage of feeding, one was euthanized and compared with the experimental group slices images. Each rat can offer two liver hyperspectral images, and in our study, one of them was randomly selected for tumor analysis. The pathological liver sections were provided by pathologists (Department of Oncology, Longhua Hospital Affiliated to Shanghai University of Traditional Chinese Medicine) collaborating with our laboratory.

We utilize the MHSI systems to capture experimental images in two different formats. One is true color images captured by an ordinary optical microscope that is doubled in magnification. The liver tissue slices are rendered as purple-red under the action of HE staining. This dataset will be compared with the results of pseudocolor synthesis after image classification. The other is hyperspectral grayscale images with wavelengths from 550 to $1000 \mathrm{~nm}$, which is stored in 60 wavebands with 2- to 6-nm spectral resolutions. To acquire and determine valuable liver tumor statistics, the liver biopsy experimental images were collected every 4 from 8 weeks until 20 weeks.

The quality of liver tissue hyperspectral grayscale images has been significantly improved after preprocessing by spectraldomain normalization and spatial-domain enhancement. Adopting a standard rat image [Figs. 6(a)-6(c)] and four experimental images at different stages [Figs. 6(d)-6(o)] as examples, the first column of Fig. 6 is the original image acquired. There are impurities such as salt and pepper noise and data artifacts in the image due to MHSI systematic errors, from which it is still impossible to extract useful information for analysis. The second column is the hyperspectral grayscale images at the $770 \mathrm{~nm}$ (The image contrast and visual effects are ideal at this wavelength.) after preprocessing. The gray value gradient of Fig. 6(b) in normal rat image and (e) in the 8-week image has been initially processed, while they are ambiguous for identifying pathological liver tissue and normal components directly. Whereas the tumor structure, the black region with lower gray value, can be pointed out by the judgment of the gray gradient in images (h), (k), and (n), which are corresponding to the liver pathological sections in 12th, 16th, and 20th week. There are no tumor components in normal rat image, Fig. 6(c) shows a spectral curve of normal liver tissue. Figures 6(f), 6(i), 6(1), and 6(o) are the spectral curves for all wavelengths at two sample points in the left hyperspectral grayscale images, in which the green spectrum expresses pathological liver tissues and the yellow spectrum represents nonpathological areas; It can be observed that for the normal tissue, the valley of the spectral curves at each stage is $\sim 0.8$. However, the spectral characteristics of the tumor components and normal tissue regions differ significantly in the hyperspectral images over a more extended period of time, and as the time period increases, the gap between the two curves gradually widens in the range of 600 to $800 \mathrm{~nm}$. The spectrum within this wavelength range is most conducive to experimental research. However, in the early (8 weeks) experimental images, the tissue of the suspected cancerous or the initial lesion is relatively similar to the nonpathological component in the reflection and absorption of electromagnetic waves, and it can be observed from Fig. 6(f) that they are very close in spectral profile, with a maximum difference of $<0.1$ at $700 \mathrm{~nm}$.

Spectral curves in different positions of liver tissue indicate that hyperspectral images can indeed be advantageous in the pathological diagnosis of tumors. The purpose of this study is further to take full use of the superior spectral properties of the hyperspectral image, to extract tumors from the early pathological sections of liver tissue, to explore the possibility of liver tumors early diagnosis, and to provide a potential method for pathological diagnosis.

\subsection{Segmentation and Classification Results}

The MWA regards a grayscale image as an elevation image, and the gradient change in the grayscale is the topographic relief. When the segmentation threshold is too high, the segmentation results of the liver pathological hyperspectral image are not accurate enough to divide the different components of the image. As the watershed segmentation algorithm is based on the spatial information of the image, the processed result is still a full wavelength (550 to $1000 \mathrm{~nm}$ ) hyperspectral image, only the grayscale image in each wavelength is distinguished into a number of small regions with locally identical label values. Taking a 20-week experimental image as an example, as shown in sample Fig. 7, (a) is the preprocessed hyperspectral grayscale image of liver tissue at $770 \mathrm{~nm}$ and (b) is the representative MWA segmentation result. An enlarged view of hepatic fibrosis is shown in Figs. 7(e)-7(h) corresponding to a square area in (a). Liver tumors are reflected in dark colors with lower gray values, whereas other areas are grayish [Fig. 7(f)].

The SVM algorithm is employed as a supervised learning classifier to recognize and classify the segmentation results. We applied spectral library information and determined spectral properties to define training examples, a portion of liver tumor and nonpathological pixels are picked at different locations in the segmentation result severally. The parameters of all wavelengths are used for testing the examples; the classification results export one-wavelength image as delineated in Fig. 7(c), and green and yellow point out the liver tumors and normal tissues, respectively. However, there are some misclassified small plaques in the region of nonpathological tissue as marked out in the red ellipses in Fig. 7(g), these plaques were confirmed by pathologists. The majority analysis tool eliminates small plaques and preserves liver tumor compositions availability as Fig. 7(h) shows. Combined with the processed results of MWA-based SVM, the eventual classification results reflect the tumor status of the pathological liver sections relatively accurate. 
Wang and Li: Quantitative analysis of liver tumors at different stages using microscopic hyperspectral...

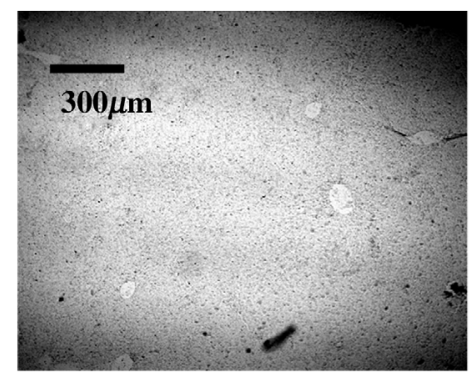

(a)

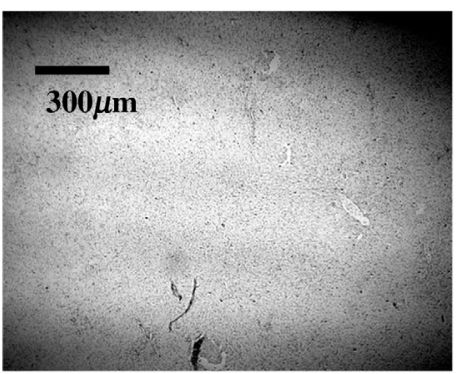

(d)

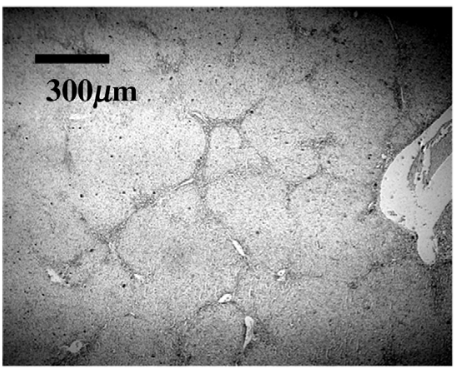

(g)

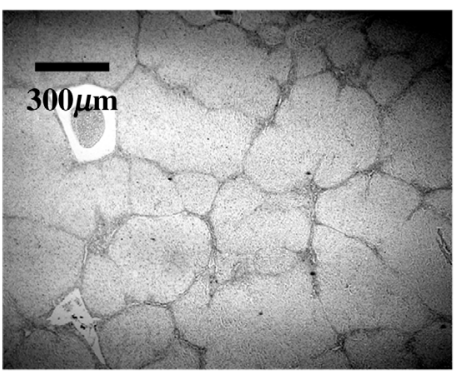

(j)

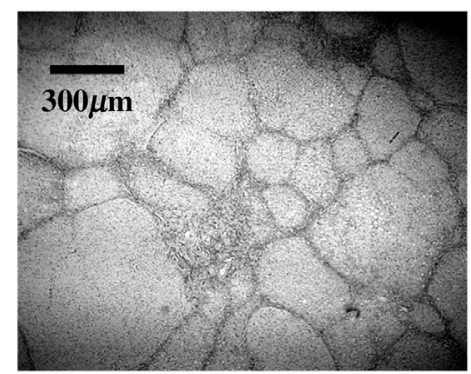

(m)

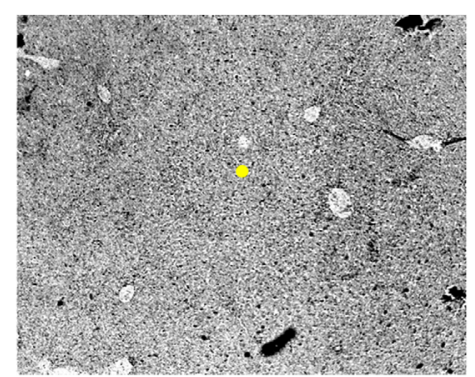

(b)

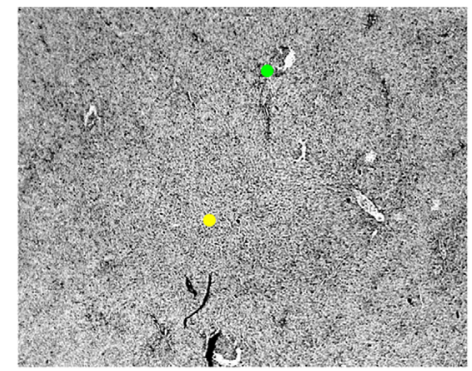

(e)

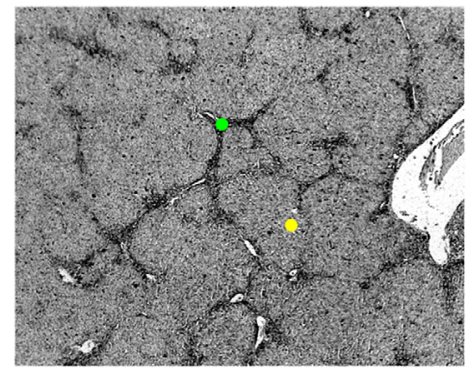

(h)

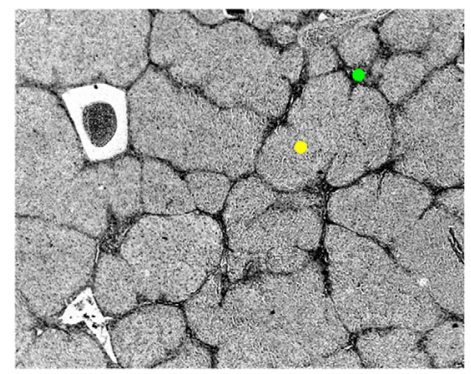

(k)

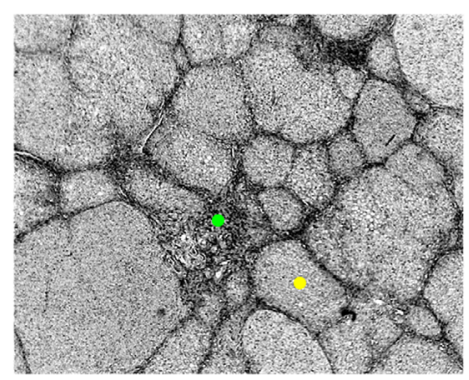

(n)

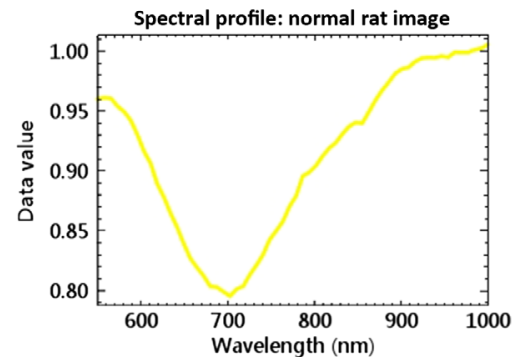

(c)

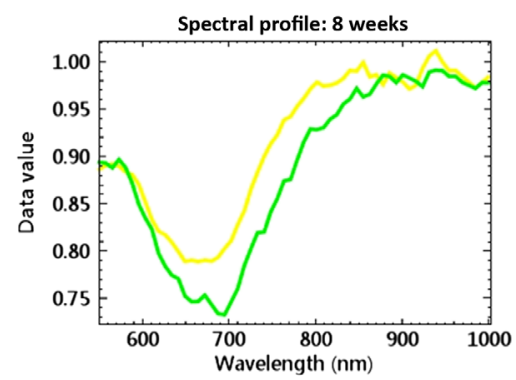

(f)

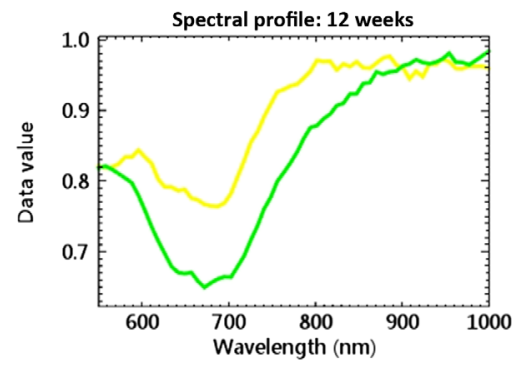

(i)

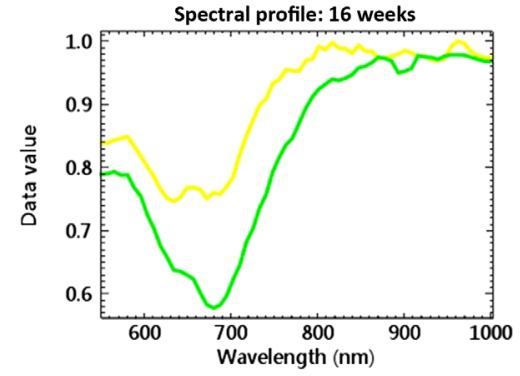

(I)

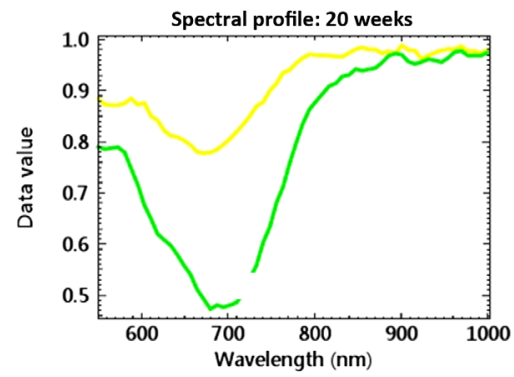

(o)

Fig. 6 Single-band images at $770 \mathrm{~nm}$ and spectra. (a) Original liver hyperspectral images of a normal rat; $(\mathrm{d}),(\mathrm{g}),(\mathrm{j})$, and $(\mathrm{m})$ original hyperspectral images of tumor-bearing rats at four experimental stages; (b), (e), (h), (k), and (n) preprocessed single-band images corresponding to the first column; (c) spectral curve corresponding to the yellow point in (b); (f), (i), (l), and (o) spectral curves of the sample points corresponding to preprocessed images. The green area represents the pathological component, and the yellow area represents the nonpathological component. 


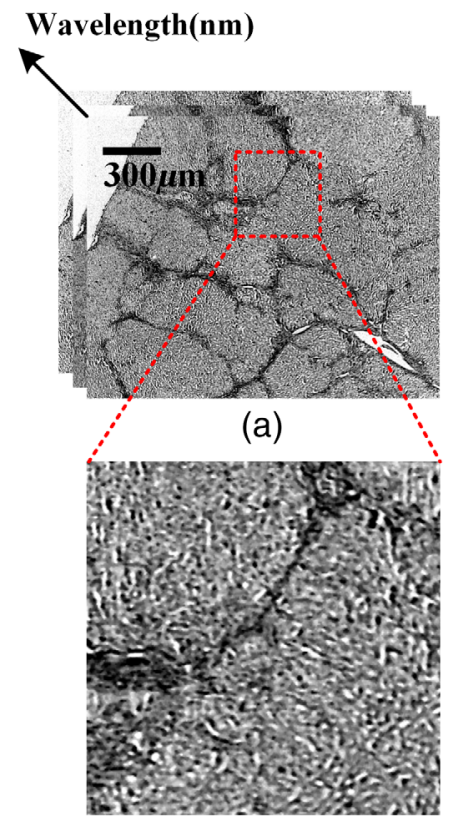

(e)

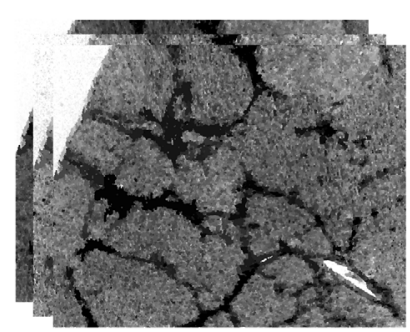

(b)

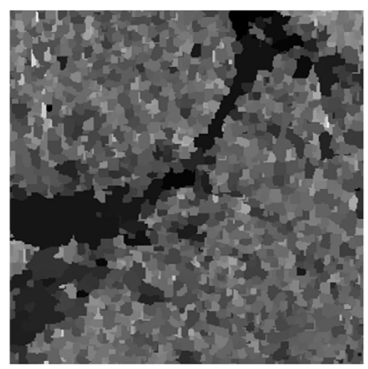

(f)

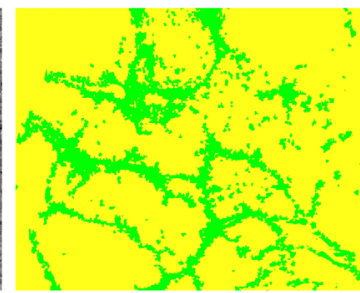

(c)

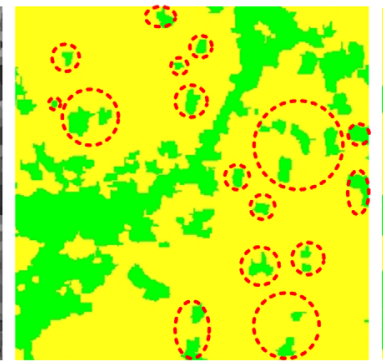

(g)

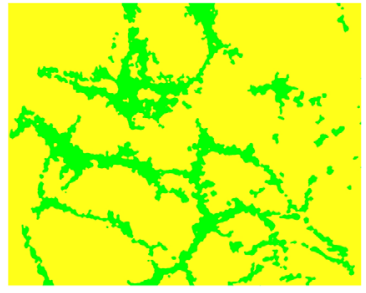

(d)

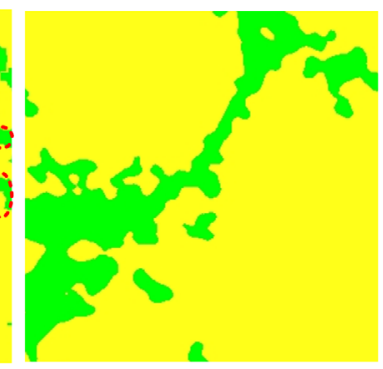

(h)

Fig. 7 An example of the processed results. (a) Preprocessed single-band image, (b) MWA segmentation image, (c) MWA-based SVM classification image, (d) postclassification image, (e), (f), (g), and (h) an enlarged view corresponding to the square areas in $(a-d)$. The red ellipses in $(g)$ are misclassified small plaques.

Using the above algorithms to process the experimental images of liver tumor samples in normal rat and at four different stages, the classification results [Figs. 8(f), 8(k), and 8(p)] of normal rat image in control group were monochrome (black), meaning that no pathological components were identified; the processed results in the experimental group are obtained as shown in Figs. 8(g)-8(t). For comparison, the standard reference images of the liver tumor provided by the pathologist are located in the second row of Figs. 8(f)-8(j). In addition, the third row of Figs. 8(k)-8(o) lists the processed results only using the SVM algorithm. Assessment criteria for liver tumor changes are given by pathologists. ${ }^{34}$ In the 8 -week hyperspectral image (b), it is difficult for the human eye to identify obvious abnormalities. The gray gradient is various, and the dark pixels are extremely dispersed, which is consistent with the early liver pathological condition. The classification results (g) performed by pathologists and the results (l) and (q) obtained by our method show that the rats' livers had undergone minor lesions at 8 weeks. In the 12-week images (h), (m), and (r), a large number of hepatocytes in cancerous lesions are accumulated, and the distribution is still relatively disordered. The abnormal hyperplasia of connective tissue in the liver causes liver fibrosis to appear initially. When the liver tissue lesions develop to 16 weeks (d), (i), (n), and (s), a large number of fibroplasias [Fig. 10(k)] and some bile duct carcinomas have developed, the fibrous structure tends to be complete; the boundaries of the segmented hepatic lobule are particularly clear. Severe hepatic fibrosis develops into cirrhosis or malignant neoplasms, resulting in the appearance of large necrotic tissue, just as the 20-week images (e), (j), $(\mathrm{o})$, and $(\mathrm{t})$. Comparing the three results, it can be seen that the approach proposed in this paper is closest to the standard reference image.

To assess the accuracy of the classification results, we utilized the confusion matrix to calculate the OA and Kappa coefficient and obtained the data shown in Table 1. The calculated Kappa coefficients often fall between 0 and 1 and can be divided into five groups to indicate the consistency of the relationship: slight (0.0 to 0.20$)$, fair $(0.21$ to 0.40$)$, moderate (0.41 to 0.60$)$, substantial ( 0.61 to 0.80$)$, and almost perfect ( 0.81 to 1 ). Table 1 shows that the OA of the SVM algorithm classification reaches $>80 \%$, but the kappa coefficient is generally $<0.6$, especially for the 8 th-week image, the OA is almost equal to our result, yet the kappa coefficient is only 0.2996, indicating that the classification consistency is weak. The OA of our method is $92.38 \%, 95.10 \%, 95.40 \%$, and $95.54 \%$, respectively, and the kappa coefficients are $>0.8$, corresponding to 8th, 12th, 16 th, and 20th week. In the middle-later stages, our processing can extract liver tumor characteristics relatively accurately and prepare for classification statistics and pseudocolor synthesis. The identification results of liver pathology sections at each time point manifest that these operations are likely to contribute a specific reference value for the early diagnosis of liver tumors.

\subsection{Liver Tumor Statistics and Pseudocolor Synthesis}

In this section, the percent tumor area in liver samples from the classification results is quantified and statistically analyzed based on the number of classified pixels. At the same time, composite pseudocolor images are generated based on the wavelength combination. The statistical results show that the percent tumor area overall follows a significant pattern: as the time period increases, the pathological morphology of the liver tissue gradually changes, and the percent tumor area expands. Table 2 shows the statistical results of the samples in this work, and 10 experimental samples and 1 control sample at each time point are used for analysis. 


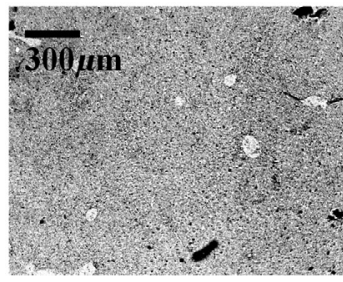

(a)

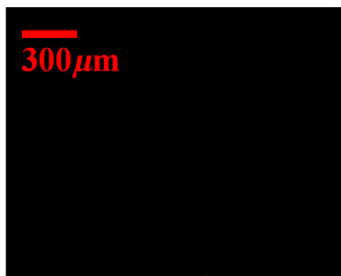

(f)

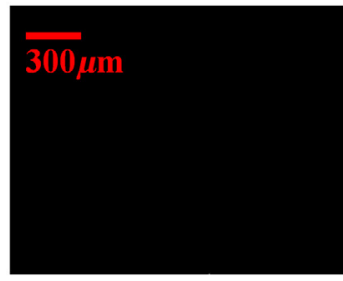

(k)

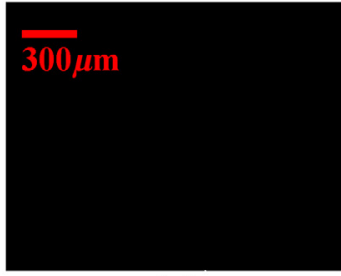

(p)

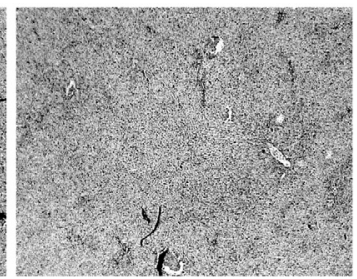

(b)

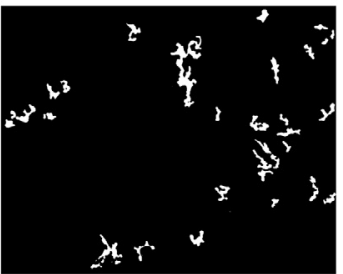

(g)

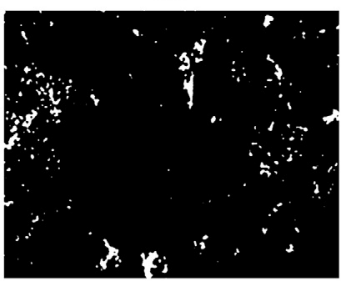

(I)

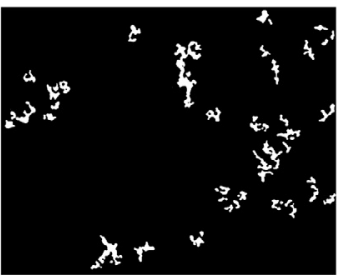

(q)

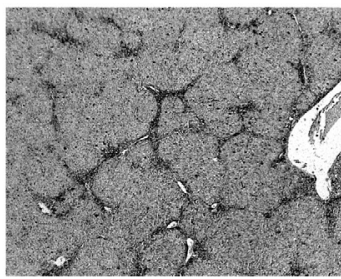

(c)

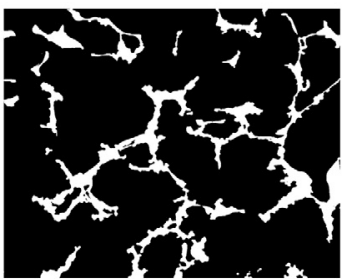

(h)

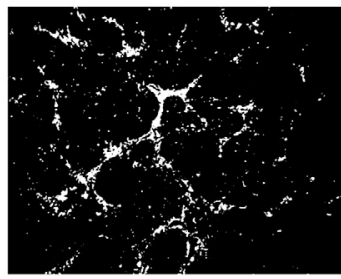

(m)

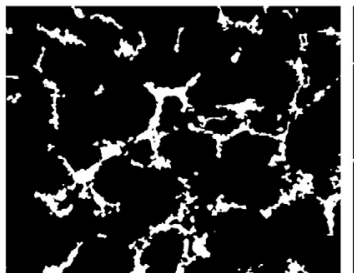

(r)

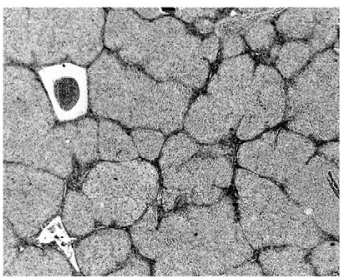

(d)

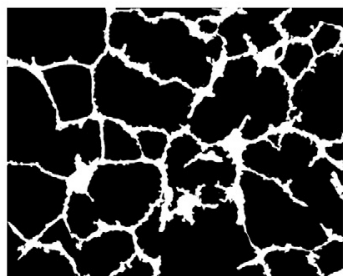

(i)

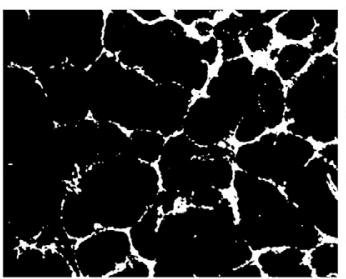

(n)

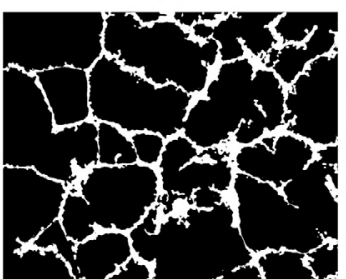

(s)

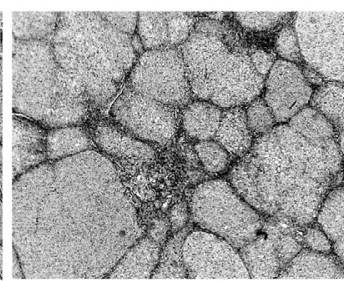

(e)

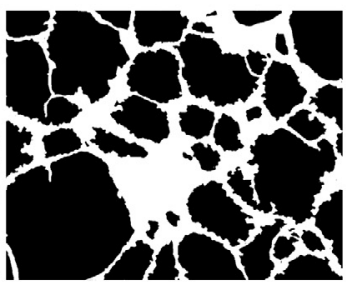

(j)

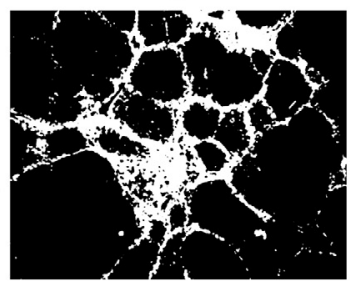

(o)

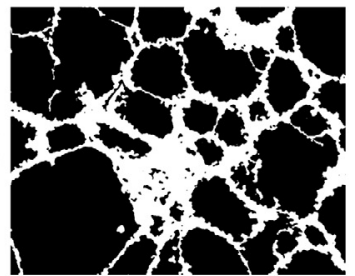

(t)

Fig. 8 The processed results at the four different time points. (a)-(e) Single-band images at $770 \mathrm{~nm}$, (f)-(j) standard classification images, (k)-(o) SVM-based results, and (p)-(t) results of the proposed method.

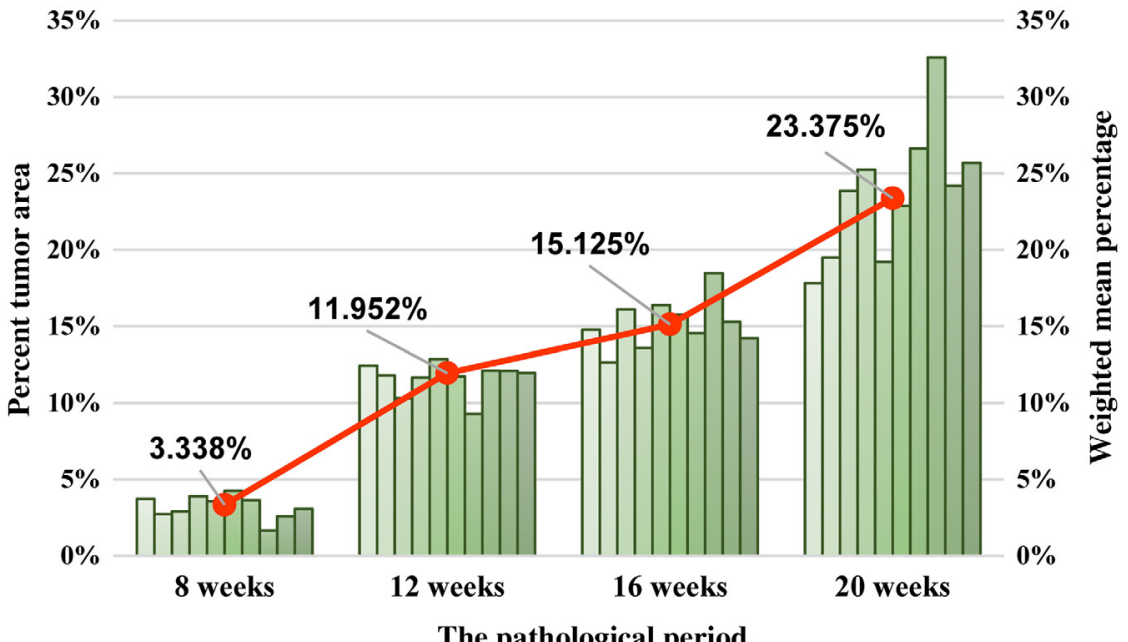

Fig. 9 The distribution of percent tumor area in liver samples.

For the data of each stage, the weighted mean is calculated according to the number of occurrences within $1 \%$ of the variation, which obtained $3.338 \%$ for 8 weeks, $11.952 \%$ for 12 weeks, $15.125 \%$ for 16 weeks, and $23.375 \%$ for 20 weeks, independently. To vividly discuss the quantification trend of tumors, Fig. 9 plots bar graphs and line graphs showing the distribution of percent tumor area in the liver samples in accordance with the data shown in Table 2. The left main axis and 


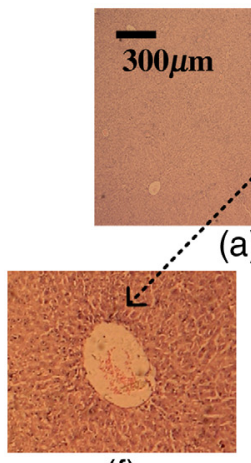

(f)

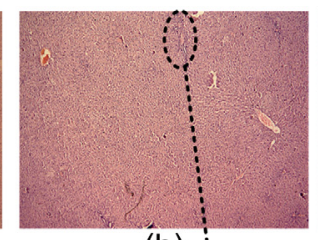

(b)

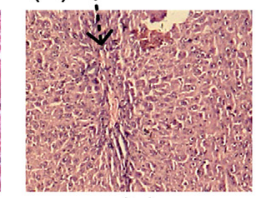

(h)

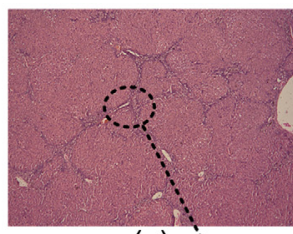

(c)

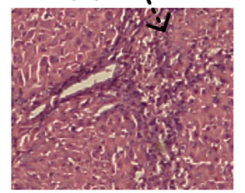

(i)

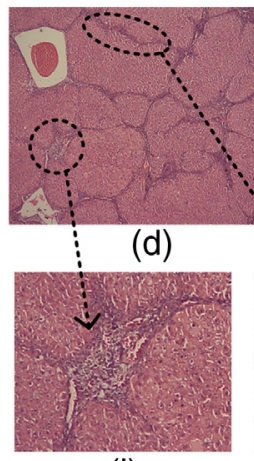

(j)

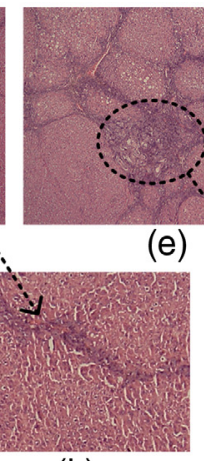

(k) (e)

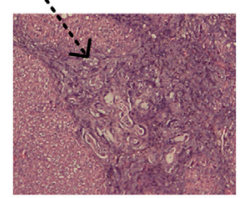

(l)

Fig. 10 Histopathological structure of bile duct carcinoma at different stages. (a)-(e) Original true color images in normal rat, 8, 12, 16, and 20 weeks, (f) normal liver lobule, (g) normal portal area (taken from other normal rat sample), (h) false lobule, (i) deformed central vein, (j) connective tissue hyperplasia in the portal area, (k) fibroplasia, and (I) intestinal type cholangiocarcinoma.

Table 1 The comparison of classification performance with different methods.

\begin{tabular}{|c|c|c|c|c|c|}
\hline Method & $\frac{\text { Time point }}{\text { Indices }}$ & $\begin{array}{c}8 \\
\text { weeks }\end{array}$ & $\begin{array}{c}12 \\
\text { weeks }\end{array}$ & $\begin{array}{c}16 \\
\text { weeks }\end{array}$ & $\begin{array}{c}20 \\
\text { weeks }\end{array}$ \\
\hline \multirow[t]{2}{*}{ SVM } & $\mathrm{OA}(\%)$ & 92.32 & 80.39 & 80.97 & 85.06 \\
\hline & Kappa & 0.2996 & 0.3961 & 0.5688 & 0.5997 \\
\hline \multirow{2}{*}{$\begin{array}{l}\text { Example-based } \\
\text { feature extraction }\end{array}$} & $\mathrm{OA}(\%)$ & 92.38 & 95.10 & 95.40 & 95.54 \\
\hline & Kappa & 0.8257 & 0.8712 & 0.9197 & 0.9162 \\
\hline
\end{tabular}

Table 2 Liver tumor classification statistics (the percent tumor area in the classification results).

\begin{tabular}{lcccc}
\cline { 1 - 3 } Time point & & & & \\
\cline { 1 - 3 } Sample & 8 weeks & 12 weeks & 16 weeks & 20 weeks \\
\cline { 1 - 4 } group (\%) & 3.725 & 12.441 & 14.781 & 17.823 \\
& 2.734 & 11.803 & 12.653 & 19.496 \\
& 2.906 & 10.313 & 16.103 & 23.857 \\
& 3.897 & 11.666 & 13.582 & 25.242 \\
& 3.573 & 12.864 & 16.386 & 19.218 \\
& 4.257 & 11.737 & 15.767 & 22.874 \\
& 3.641 & 9.284 & 14.558 & 26.630 \\
& 1.658 & 12.114 & 18.473 & 32.582 \\
& 2.589 & 12.103 & 15.297 & 24.187 \\
& 3.075 & 11.966 & 14.221 & 25.691 \\
Control group (\%) & 0 & 0 & 0 & 0 \\
Weighted mean (\%) & 3.338 & 11.952 & 15.125 & 23.375 \\
\hline
\end{tabular}

the series of green bars explain the percent tumor area for each experimental time point, and the right secondary axis and the red polyline reveal the weighted mean of the datasets, which changes over time. As discussed above, in the early hyperspectral images of pathological liver sections, it is difficult to identify and extract the tumor components, and the difference in the degree of tumor lesions is not entirely visible. From the table and diagram, it can also be concluded that liver tumors develop faster after 8 weeks, and in the development of later stages, the extent of liver tumors has begun to diversify. For example, in a few of 16-week liver tissue hyperspectral images, the percent tumor area is higher than that in the 20 -week images. In addition, in the set of experimental data from the same time point, the variation among samples is even $>10 \%$; tumor lesions in some of the 20-week images cover a wide range of areas, such as nearly one-third of the liver tissue section. In contrast, we did not detect any tumor components in the control rat samples at the four time points.

The tracking assessments and quantitative statistics of tumors at four different stages further demonstrate the possible significance of hyperspectral imaging in prediagnosis of liver tumors. Although the statistical data in the above diagrams offer some information about the lesion status of liver tumors, it lacks visuality. In the clinical diagnosis of liver tumors, pathologists prefer to observe image data, and analyzing the pathological structure of liver tumor is an indispensable part for diagnosis. In practical applications, the pathological section images collected via the optical microscope are processed with the dyeing agent, but the displayed colors are relatively dull, the interesting areas are not prominent, and the amount of information is relatively small as shown in the first column of Fig. 10. Especially in the 8-week true color image (b), the presence of normal hepatic lobules and liver cells can be inspected in partial tissue sections, but almost for all the sections, the human eye can hardly directly distinguish the location of the liver tumors.

The pseudocolor synthesis method based on wavelength combination can solve the problem of true color images. The maximum difference in standard deviation occurs approximately in the vicinity of 700 and $900 \mathrm{~nm}$ and the longer the distance between the combined wavelengths, the smaller the minimum sum of correlation coefficients according to Fig. 5(b). The experimental results show that the wavelengths at 700 and $900 \mathrm{~nm}$ of the preprocessed hyperspectral grayscale image are selected as the pseudocolor synthesized $\mathrm{R}$ and $\mathrm{B}$ channels, and 
the image of the classification result as the G channel, the optimal synthetic effect can be obtained. The third column in Fig. 11 exhibits the eventually experimental results of the four stages, in which the dark blue area is the protruding part of the liver tumors, and the noncancerous one is synthesized as greenish, resulting in enhanced visual contrast. The greenish regional compositions will be the direction of our next research. The strong chromatic aberration is more advantageous to observe the morphological change of tumor. Figures 11(a)-11(c) show the normal rat images in the control group; only the normal tissues like liver lobule and portal area can be found in the pseudocolor composite images. In the early stage, the distribution of liver cells is scattered, and the convergent nuclear color of the liver cells is slightly deepened as shown in Fig. 11(f), meanwhile, the central vein has not yet shifted, both in quantity and volume are small. In the medium stages, many false lobules are appearing in the liver tissue, which is the characteristic lesion of cirrhosis and tumor. The false lobules [Fig. 10(h)] are hyperplasias of hepatic cell masses formed by the proliferation of fibrous tissue separation and entrapment, with a disordered arrangement of hepatic cords. The hyperplasia of connective tissues emerges in the portal area [Fig. 10(j)], and the central vein appears multiple and deformed [Fig. 10(i)], which are shown in Figs. 11(i) and 11(1). Referring to Fig. 11(o), the characteristics of the tumors in the pathological liver image at 20 weeks have been clearly highlighted, accompanied by hepatocyte steatosis, necrotic structures, namely, a large number of connective tissue hyperplasia in the portal area, dysplasia of bile duct cells, and

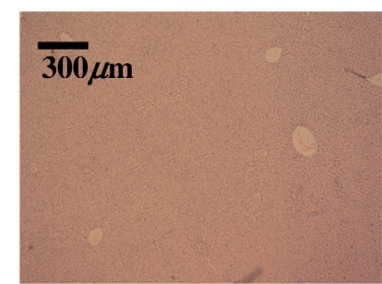

(a)

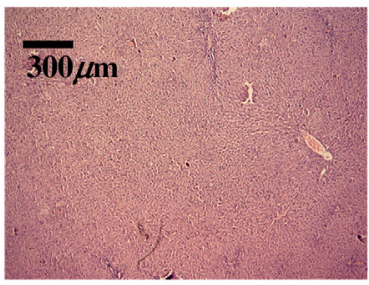

(d)

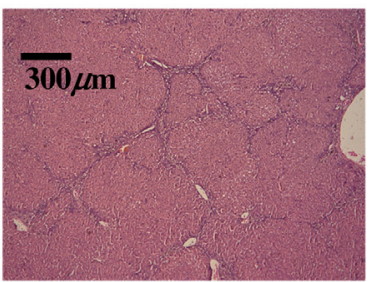

(g)

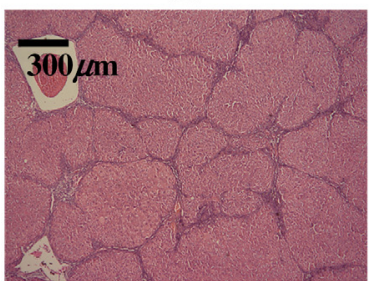

(j)

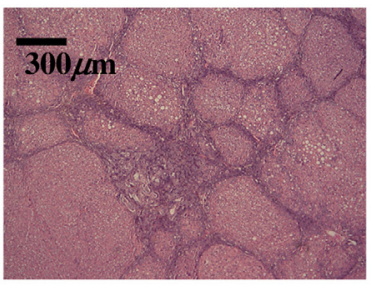

(m)

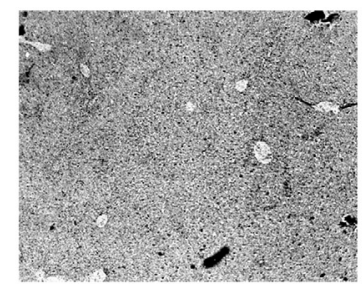

(b)

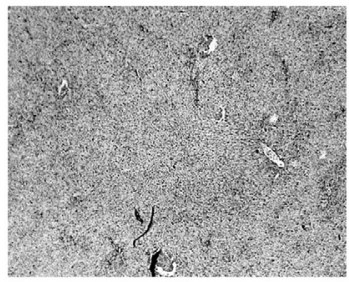

(e)

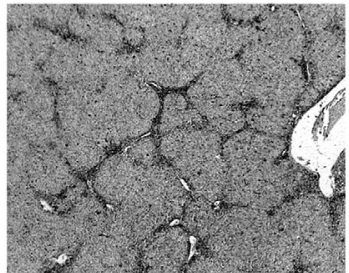

(h)

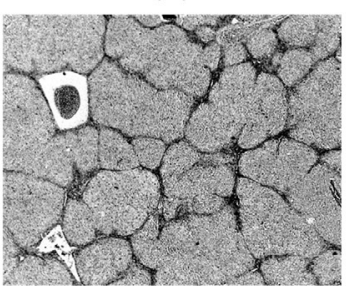

(k)

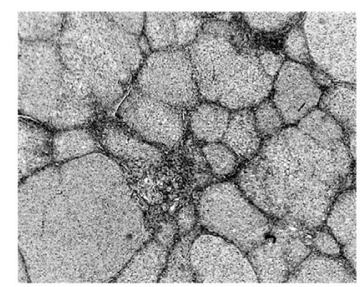

(n)

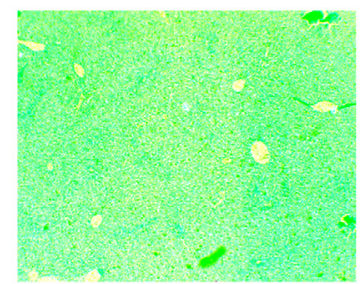

(c)

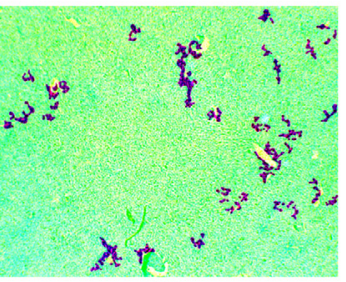

(f)

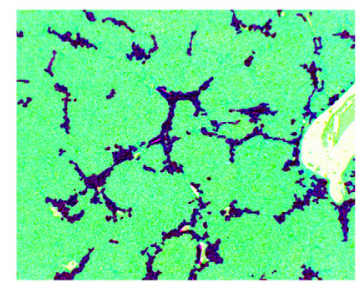

(i)

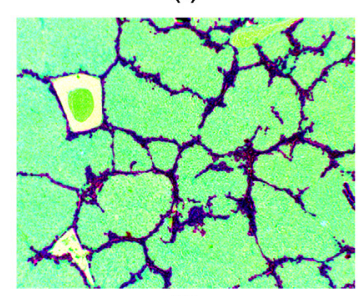

(I)

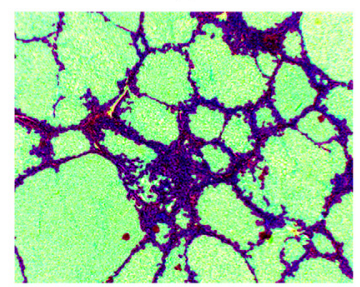

(o)

Fig. 11 Comparison of original true color (microscope) images with pseudocolor composite images at different time points. (a), (d), (g), (j), and (m) Original true color images in normal rats at $8,12,16$, and 20 weeks; (b), (e), (h), (k), and (n) corresponding single-band images; and (c), (f), (i), (l), and (o) corresponding pseudocolor composite images. 
tumor formation [Fig. 10(1)]. By comparing the true color images, single-band images, and pseudocolor composite images from the same time point, the advantage of hyperspectral images can be found. Hyperspectral images cover abundant spatial and spectral information of liver tumors, on this basis, quantitative statistics analysis affords a foundation for judging the extent of early tumors and pseudocolor composite images in the RGB color space can effectively weaken the influence of noncancerous components in liver tissues and reflect the liver lesion status.

\section{Conclusion}

The age of onset of hepatic malignancy presents a trend of youthfulness. Millions of people are diagnosed with liver disease every year and even die of liver cancer. Although at present, with the developments at the therapeutic level, the means for treating liver tumors are becoming more and more advanced. However, the timely treatment of liver tumors still poses a bottleneck in the medical field, and prediagnosis is of vital importance for controlling the deterioration of tumors. This paper provides a method that can probably be used for early diagnosis of liver tumors. Rat liver pathological sections under four different stages are utilized as experimental samples, and MHSI systems are applied to collect multiple types of images for processing and analysis. First, the pretreatment of the original image eliminates a multitude of interference factors. Second, in the liver tumor feature extraction, the MWA supplies the basis for spatial segmentation in virtue of grayscale, while the SVM algorithm combines the spectral properties of hyperspectral images for identification operation. After removing small plaques with MMA, the percent tumor area in the liver samples is calculated for quantitative analysis, and pseudocolor images are synthesized to visualize the changes in the pathological structure of liver tissues. Ultimately, the pooled results at 8 , 12,16 , and 20 weeks are $3.338 \%, 11.952 \%, 15.125 \%$, and $23.375 \%$, respectively. We compared the SVM and MWAbased SVM results with the standard classification images. The results proved the feasibility of our method. This finding suggests the potential of MHSI in the field of medical image processing, particularly in tumor prediagnosis. In later studies, more liver tissue sections in the early stage (such as 8 weeks and earlier) with suspected lesions will be used to verify the validity of the proposed method. In addition, there is no refinement of the liver tumor components in the current work, and extensive experiments are needed in the future to extract more complex liver tissue features and yield more accurate information for early diagnosis.

\section{Disclosures}

The authors have no relevant financial interests or conflicts of interest to disclose.

\section{Acknowledgments}

This work was supported by the National Natural Science Foundation of China (Grant No. 61377107), the Science and Technology Commission of Shanghai Municipality (Grant No. 18511102500). The authors wish to thank Dr. Guanzhen Yu for providing the pathological information of liver samples.

\section{References}

1. S. Chauvie et al., "Automatic liver detection and standardised uptake value evaluation in whole-body positron emission tomography/computed tomography scans," Comput. Meth. Programs Biomed. 156, 47-52 (2018).

2. B. Kim et al., "Imaging findings of primary hepatic angiosarcoma on gadoxetate disodium-enhanced liver MRI: comparison with hepatic haemangiomas of similar size," Clin. Radiol. 73(3), 244-253 (2018).

3. S. Y. Song et al., "Celiac axis and common hepatic artery variations in 5002 patients: systematic analysis with spiral CT and DSA," Radiology 255(1), 278-288 (2010).

4. S. Shimada et al., "Macro- and microscopic findings of ICG fluorescence in liver tumors," World J. Surg. Oncol. 13, 198 (2015).

5. J. Zhou et al., "Stage scoring of liver fibrosis using Mueller matrix microscope," Proc. SPIE 10024, 100240M (2016).

6. D. S. Ashour et al., "Hybrid feature extraction techniques for microscopic hepatic fibrosis classification," Microsc. Res. Tech. 81(3), 338-347 (2018).

7. L. Moraru et al., "Texture analysis of parasitological liver fibrosis images," Microsc. Res. Tech. 80(8), 862-869 (2017).

8. N. Giannakeas et al., "A clustering based method for collagen proportional area extraction in liver biopsy images," Conf. Proc. IEEE Eng. Med. Biol. Soc. 2015, 3097-3100 (2015).

9. M. G. Tsipourasa et al., "A methodology for automated CPA extraction using liver biopsy image analysis and machine learning techniques," Comput. Meth. Programs Biomed. 140, 61-68 (2017).

10. D. C. Tsouros et al., "Automated collagen proportional area extraction in liver biopsy images using a novel classification via clustering algorithm," in 2017 IEEE 30th Int. Symp. on Computer-Based Medical Systems (CBMS), pp. 30-34 (2017).

11. Q. L. Li et al., "Review of spectral imaging technology in biomedical engineering: achievements and challenges," J. Biomed. Opt. 18(10), 100901 (2013).

12. A. Jarman et al., "Hyperspectral microscopy and cluster analysis for oral cancer diagnosis," Proc. SPIE 10076, 100761I (2017).

13. N. Neittaanmaki-Perttu et al., "Delineating margins of lentigo maligna using a hyperspectral imaging system," Acta Derm.-Venereol. 95(5), 549-552 (2015).

14. N. Neittaanmaki-Perttu et al., "Detecting field cancerization using a hyperspectral imaging system," Lasers Surg. Med. 45(7), 410-417 (2013).

15. E. Hashimoto et al., "Tissue classification of liver pathological tissue specimens image using spectral features," Proc. SPIE 10140, 101400Z (2017).

16. J. Irigoyen and J. Herraez, "Electromagnetic spectrum and color vision," in ISPA 2004. Proc. of the 3rd Int. Symp. on Image and Signal Processing and Analysis (IEEE Cat. No. 03EX651), Vol. 622, pp. 626-629 (2003).

17. G. L. Lu and B. W. Fei, "Medical hyperspectral imaging: a review," J. Biomed. Opt. 19(1), 010901 (2014).

18. Q. L. Li et al., "AOTF based molecular hyperspectral imaging system and its applications on nerve morphometry," Appl. Opt. 52(17), 3891-3901 (2013).

19. G. Yunfeng et al., "AOTF based molecular hyperspectral imaging system and its image pre-processing method," in Proc. of 8th Int. Conf. on Biomedical Engineering and Informatics (BMEI), pp. 14-18 (2015).

20. S. Esakkirajan et al., "Removal of high density salt and pepper noise through modified decision based unsymmetric trimmed median filter," IEEE Signal Process. Lett. 18(5), 287-290 (2011).

21. R. H. Chan, C. W. Ho, and M. Nikolova, "Salt-and-pepper noise removal by median-type noise detectors and detail-preserving regularization," IEEE Trans. Image Process. 14(10), 1479-1485 (2005).

22. L. Vincent and P. Soille, "Watersheds in digital spaces: an efficient algorithm based on immersion simulations," IEEE Trans. Pattern Anal. Mach. Intell. 13(6), 583-598 (1991).

23. Y. Tarabalka, J. Chanussot, and J. A. Benediktsson, "Segmentation and classification of hyperspectral images using watershed transformation," Pattern Recognit. 43(7), 2367-2379 (2010).

24. J. B. Jiang, X. J. Qiao, and R. Y. He, "Use of near-infrared hyperspectral images to identify moldy peanuts," J. Food Eng. 169, 284-290 (2016).

25. R. S. Majdar and H. Ghassemian, "Spectral-Spatial classification of hyperspectral images using functional data analysis," Remote Sens. Lett. 8(5), 488-497 (2017).

26. M. Golipour, H. Ghassemian, and F. Mirzapour, "Integrating hierarchical segmentation maps with MRF prior for classification of 
hyperspectral images in a Bayesian framework," IEEE Trans. Geosci. Remote Sens. 54(2), 805-816 (2016).

27. J. L. Xu, C. Riccioli, and D. W. Sun, "Comparison of hyperspectral imaging and computer vision for automatic differentiation of organically and conventionally farmed salmon," J. Food Eng. 196, 170-182 (2017).

28. T. van Gestel et al., "Benchmarking least squares support vector machine classifiers," Mach. Learn. 54(1), 5-32 (2004).

29. J. Wang et al., "An automatic liver fibrosis qualitative analysis method based on hyperspectral images," Proc. SPIE 10806, 1080634 (2018).

30. M. Sokolova and G. Lapalme, "A systematic analysis of performance measures for classification tasks," Inf. Process. Manage. 45(4), 427-437 (2009).

31. W. Tang et al., "Kappa coefficient: a popular measure of rater agreement," Shanghai Arch. Psychiatry 27(1), 62-67 (2015).

32. J. Zhang et al., "Speech spectrogram display in pseudo-color based on non-linear transform method," J. Comput. Appl. (China) 30(1), 270-273 (2010).

33. M. Sammouda et al., "Segmentation and analysis of liver cancer pathological color images based on artificial neural networks," in Proc. of Int.
Conf. on Image Processing (Cat. 99CH36348), Vol. 393, pp. 392-396 (1999).

34. G.-F. Wang et al., "Aspirin interrupts bile duct carcinoma in rats induced by thioacetamide," Acad. J. Second Mil. Med. Univ. 39(3), 285-290 (2018).

Jiansheng Wang is an undergraduate student at Shanghai Key Laboratory of Multidimensional Information Processing, East China Normal University, Shanghai, China. She is interested in using MHSI systems to analyze pathological sections for biomedical applications.

Qingli Li received his BS and MS degrees in computer science and engineering from Shandong University, Jinan, China, in 2000 and 2003, respectively, and his $\mathrm{PhD}$ in pattern recognition and intelligent system from Shanghai Jiaotong University, Shanghai, China, in 2006. Currently, he is a professor at Shanghai Key Laboratory of Multidimensional Information Processing, East China Normal University, Shanghai, China, where he is engaged in research on medical imaging, pattern recognition, and image processing. 